\title{
Clothed particles in mesodynamics, quantum electrodynamics and other field models
}

\author{
Aleksandr Shebeko* \\ Institute for Theoretical Physics National Scientific Center "Kharkov Institute of Physics \& \\ Technology", Kharkov 61108, Ukraine \\ E-mail: Shebekodkipt.kharkov.ua
}

First, we will show how the notion of clothing in quantum field theory (QFT), put forward by Greenberg and Schweber and developed by M. I. Shirokov, can be employed not only in the theory of interacting meson and nucleon fields (see, e.g., our previous works ), but in quantum electrodynamics (QED) and, perhaps, in quantum chromodynamics (QCD) too. As before, using the instant form of relativistic dynamics and applying the method of unitary clothing transformations (shortly, UCT method) we have derived a novel analytic expression for the QED Hamiltonian in the clothed-particle representation (CPR) in which the so-called bad terms are simultaneously removed from Hamiltonian and boosts via one and the same UCT. In spite of the primary electromagnetic (EM) interaction has been chosen in the Coulomb gauge (CG) with an apparent violation of the Lorentz invariance the latter is restored owing to the cancellation of the noncovariant Coulomb interaction contribution already with the first clothing transformation. A similar cancellation has been found by us in case of the vector mesons (spin 1 bosons) interacting via the Yukawa-type couplings with the nucleons (spin 1/2 fermions). Second, we are trying to realize this notion in quantum chromodynamics (QCD) (to be definite for the gauge group $S U(3)$ ) when drawing parallels between QCD and QED. It is convenient to do it along the guideline: the well-known QCD Lagrangian density with the hermitean and traceless vector potentials, the mass and covariant derivative matrices in color space, the color-Maxwell equations and color gauge-invariant energy-momentum stress tensor versus their colorless counterparts in QED.

XXII International Baldin Seminar on High Energy Physics Problems

15-20 September, 2014

JINR, Dubna, Russia

* Speaker. 
As the motto of our research we are keeping in mind that:

"... in theories with derivative couplings or spins $j \geq 1$, it is not enough

to take Hamiltonian as the integral over space of a scalar interaction density;

we also need to add non-scalar terms to the interaction density to

compensate non-covariant terms in the propagators ..."

from Chapter IV in: S. Weinberg The Quantum Theory of Fields Vol. I, 1995.

What follows are some fragments of our explorations:

\section{Mass-changing Bogoliubov type transformations. Mass counterterms}

Our departure point is the Hamiltonian

$$
H=H(\stackrel{\circ}{\alpha})=H_{0}(\stackrel{\circ}{\alpha})+V_{0}(\stackrel{\circ}{\alpha})
$$

where unperturbed Hamiltonian $H_{0}(\stackrel{\circ}{\alpha})$ and interaction term $V_{0}(\stackrel{\circ}{\alpha})$ depend on the creation and destruction operators of "bare" particles with unphysical masses and coupling constants. Here, $\stackrel{\circ}{\alpha}$ denotes the set of all these operators. For example, in case of a spinor (fermion) field $\psi$ and a neutral pseudoscalar meson field $\phi$ one has to introduce operators $\stackrel{\circ}{a}(\mathbf{k}), \stackrel{\circ}{b}(\mathbf{p}, r), \stackrel{\circ}{d}(\mathbf{p}, r)$ and their adjoint counterparts, respectively, for mesons, nucleons and antinucleons. One has in the Schrödinger $(S)$ picture

$$
\begin{gathered}
\phi(\mathbf{x})=(2 \pi)^{-3 / 2} \int d \mathbf{k}\left(2 \omega_{\mathbf{k}}^{\circ}\right)^{-1 / 2}[\stackrel{\circ}{a}(\mathbf{k})+\stackrel{\circ}{a} \dagger(-\mathbf{k})] \exp (i \mathbf{k x}), \\
\psi(\mathbf{x})=\int d \mathbf{p}\left(m_{0} /(2 \pi)^{3} E_{\mathbf{p}}^{\circ}\right)^{1 / 2}\left[\stackrel{\circ}{u}(\mathbf{p} r) \stackrel{\circ}{b}(\mathbf{p} r)+\stackrel{\circ}{v}(-\mathbf{p} r) \stackrel{\circ}{d}^{\dagger}(-\mathbf{p} r)\right] \exp (i \mathbf{p x}),
\end{gathered}
$$

where $\mathbf{k}, \mathbf{p}$ and $r$ are the particle momenta and the fermion polarization index, two Dirac spinors $\stackrel{\circ}{u}$ and $\stackrel{\circ}{v}$ satisfy the conventional equations $\left(\hat{p}^{\circ}-m_{0}\right) \stackrel{\circ}{u}(\mathbf{p}, r)=0$ and $\left(\hat{p}^{\circ}+m_{0}\right) \stackrel{\circ}{v}(\mathbf{p}, r)=0$ with $\hat{p}^{\circ}=E_{\mathbf{p}}^{\circ} \gamma^{0}-\mathbf{p} \gamma$, energies $E_{\mathbf{p}}^{\circ}=\sqrt{\mathbf{p}^{2}+m_{0}^{2}}$ and $\omega_{\mathbf{k}}^{\circ}=\sqrt{\mathbf{k}^{2}+\mu_{0}^{2}}$, the unknown values $m_{0}$ and $\mu_{0}$ play role of the bare (nonrenormalized) masses.

As usually,

$$
\begin{aligned}
{\left[\stackrel{\circ}{a}(\mathbf{k}), \stackrel{\circ}{a}^{\dagger}\left(\mathbf{k}^{\prime}\right)\right] } & =\delta\left(\mathbf{k}-\mathbf{k}^{\prime}\right), \\
\left\{\stackrel{\circ}{b}(\mathbf{p}, r), \stackrel{\circ}{b}^{\dagger}\left(\mathbf{p}^{\prime}, r^{\prime}\right)\right\} & =\left\{\stackrel{\circ}{d}(\mathbf{p}, r), \stackrel{\circ}{d}\left(\mathbf{p}^{\prime}, r^{\prime}\right)\right\}=\delta_{r r^{\prime}} \delta\left(\mathbf{p}-\mathbf{p}^{\prime}\right) .
\end{aligned}
$$

The corresponding unperturbed Hamiltonian is

$$
H_{0}(\stackrel{\circ}{\alpha})=\int d \mathbf{k} \omega_{\mathbf{k}}^{\circ} \stackrel{\circ}{a}^{\dagger}(\mathbf{k}) \stackrel{\circ}{a}(\mathbf{k})+\int d \mathbf{p} E_{\mathbf{p}}^{\circ}\left[\stackrel{\circ}{b}^{\dagger}(\mathbf{p}, r) \stackrel{\circ}{b}(\mathbf{p}, r)+\stackrel{\circ}{d}^{\dagger}(\mathbf{p}, r) \stackrel{\circ}{d}(\mathbf{p}, r)\right] .
$$

Now, let us consider a set $\alpha=\left(a, a^{\dagger}, \ldots\right)$ of the destruction and creation operators for particles with given (not obligatorily observable) masses, e.g., masses of constituent quarks. If $m$ and $\mu$ take on 
some physical values, such a representation refers to "the bare particles with physical masses" (see []]-[]] $)$. By definition, the operators $\alpha$ enter

$$
\begin{gathered}
\phi(\mathbf{x})=\int d \mathbf{k}\left(2(2 \pi)^{3} \omega_{\mathbf{k}}\right)^{-1 / 2}\left[a(\mathbf{k})+a^{\dagger}(-\mathbf{k})\right] \exp (i \mathbf{k} \mathbf{x}), \\
\psi(\mathbf{x})=\int d \mathbf{p}\left(m /(2 \pi)^{3} E_{\mathbf{p}}\right)^{1 / 2} \sum_{\mu}\left[\bar{u}(\mathbf{p} \mu) b(\mathbf{p} \mu)+v(-\mathbf{p} \mu) d^{\dagger}(-\mathbf{p} \mu)\right] \exp (i \mathbf{p} \mathbf{x}) .
\end{gathered}
$$

By doing so, we find links

$$
\frac{\stackrel{\circ}{a}(\mathbf{k})+\stackrel{\circ}{a} \dagger(-\mathbf{k})}{\sqrt{\omega_{\mathbf{k}}^{\circ}}}=\frac{a(\mathbf{k})+a^{\dagger}(-\mathbf{k})}{\sqrt{\omega_{\mathbf{k}}}}, \forall \mathbf{k},
$$

and so on.

Moreover, since the operators $\alpha$ are assumed to meet the same commutation rules as the operators $\stackrel{\circ}{\alpha}$ do, it allows us to connect them by a similarity transformation

$$
\stackrel{\circ}{a}(\mathbf{k})=T a(\mathbf{k}) T^{\dagger}, \stackrel{\circ}{b}(\mathbf{p}, r)=T b(\mathbf{p}, r) T^{\dagger}, \stackrel{\circ}{d}(\mathbf{p}, r)=T d(\mathbf{p}, r) T^{\dagger}
$$

with $T=T_{m e s} \otimes T_{f e r m}$, where, e.g.,

$$
T_{m e s}=\exp \left[-\frac{1}{2} \int d \mathbf{k} \chi_{k}\left(a^{\dagger}(\mathbf{k}) a^{\dagger}(-\mathbf{k})-a(\mathbf{k}) a(-\mathbf{k})\right)\right],
$$

with $\sqrt{\omega_{\mathbf{k}}} \exp \chi_{k}=\sqrt{\omega_{\mathbf{k}}^{\circ}}$. Then we get

$$
\stackrel{\circ}{a}(\mathbf{k})=\cosh \chi_{k} a(\mathbf{k})+\sinh \chi_{k} a^{\dagger}(-\mathbf{k}),
$$

where

$$
\begin{aligned}
& \cosh \chi_{k}=\frac{1}{2}\left[\sqrt{\frac{\omega_{\mathbf{k}}^{\circ}}{\omega_{\mathbf{k}}}}+\sqrt{\frac{\omega_{\mathbf{k}}^{\circ}}{\omega_{\mathbf{k}}^{\circ}}}\right], \\
& \sinh \chi_{k}=\frac{1}{2}\left[\sqrt{\frac{\omega_{\mathbf{k}}^{\circ}}{\omega_{\mathbf{k}}}}-\sqrt{\frac{\omega_{\mathbf{k}}^{\circ}}{\omega_{\mathbf{k}}^{\circ}}}\right] .
\end{aligned}
$$

Explicit expression for the operator $T_{\text {ferm }}$, that acts on the fermionic sector of the Fock space of boson-fermion states, is given in [[]], where one can find,

$$
H_{0}(\stackrel{\circ}{\alpha})=H_{F}(\alpha)+M_{\text {ren,mes }}(\alpha)+M_{\text {ren, ferm }}(\alpha),
$$

where

$$
\begin{gathered}
H_{F}(\alpha)=\int d \mathbf{k} \omega_{\mathbf{k}} a^{\dagger}(\mathbf{k}) a(\mathbf{k})+\int d \mathbf{p} E_{\mathbf{p}}\left[b^{\dagger}(\mathbf{p}, r) b(\mathbf{p}, r)+d^{\dagger}(\mathbf{p}, r) d(\mathbf{p}, r)\right], \\
M_{\text {ren,mes }}(\alpha)=\frac{\mu_{0}^{2}-\mu^{2}}{4} \int \frac{d \mathbf{k}}{\omega_{\mathbf{k}}}\left[a^{\dagger}(\mathbf{k}) a(\mathbf{k})+a(\mathbf{k}) a(-\mathbf{k})+H . c .\right],
\end{gathered}
$$

while the fermion mass counterterm has form

$$
M_{\text {ferm }}(\alpha)=m \delta m\left\{b^{\dagger} M_{11} b+b \dagger M_{12} d^{\dagger}+d M_{21} b+d^{\dagger} M_{22} d\right\},
$$


where the matrix $M$ is given by

$$
M=\left[\begin{array}{ll}
M_{11} & M_{12} \\
M_{21} & M_{22}
\end{array}\right]=\frac{\delta\left(\mathbf{p}^{\prime}-\mathbf{p}\right)}{E_{\mathbf{p}}}\left[\begin{array}{ll}
\delta_{r^{\prime} r} & \bar{u}\left(\mathbf{p}^{\prime}, r^{\prime}\right) v(-\mathbf{p}, r) \\
\bar{v}\left(-\mathbf{p}^{\prime}, r^{\prime}\right) u(\mathbf{p}, r) & \delta_{r^{\prime} r}
\end{array}\right],
$$

that is equivalent to

$$
M_{\text {ferm }}=\delta m \int d \mathbf{x} \bar{\psi}(\mathbf{x}) \psi(\mathbf{x}) .
$$

At this point, one should note that the transition (피) has much in common with the so-called coshsinh transformation for pair of boson operators, introduced by Bogoliubov ([6] and refs. therein) in the theory of many-body systems. However, such a resemblance is rather mathematical since the Bogoliubov transformation and the transformation $T_{m e s}$ have different scopes. As a matter of fact, the Bogoliubov transformation is related to the Hamiltonian of weakly interacting bosons to reduce it to diagonal form in the representation of quasiparticles . In the respect, the argument $\chi_{k}$ is determined there with the help of a completely different physical condition. Instead, in the case of the transformation (피) we deal with free bosons and move to the opposite direction: from the diagonal form of Eq. (ㄴ.5), to the form (때2) in which the number of bosons with a new mass $\mu$ is not conserved.

\section{Underlying formalism}

The UCT method is aimed at expressing a field Hamiltonian through the clothed-particle creation (annihilation) operators $\alpha_{c}$, e.g., $a_{c}^{\dagger}\left(a_{c}\right), b_{c}^{\dagger}\left(b_{c}\right)$ and $d_{c}^{\dagger}\left(d_{c}\right)$ via UCTs $W\left(\alpha_{c}\right)=W(\alpha)=$ $\exp R, R=-R^{\dagger}$ in the similarity transformation

$$
\alpha=W\left(\alpha_{c}\right) \alpha_{c} W^{\dagger}\left(\alpha_{c}\right)
$$

that connects set $\alpha$ in the BPR with new operators $\alpha_{c}$ in the CPR.

A key point of the clothing procedure is to remove the so-called bad terms from the Hamiltonian

$$
H \equiv H(\alpha)=H_{F}(\alpha)+H_{I}(\alpha)=W\left(\alpha_{c}\right) H\left(\alpha_{c}\right) W^{\dagger}\left(\alpha_{c}\right) \equiv K\left(\alpha_{c}\right) .
$$

By definition, such terms prevent the physical vacuum $|\Omega\rangle$

(the $H$ lowest energy eigenstate) and the one-clothed-particle states $|n\rangle_{c}=a_{c}^{\dagger}(n)|\Omega\rangle$

to be the $H$ eigenvectors for all $n^{1}$ included. Bad terms occur every time when any normally ordered product

$$
a^{\dagger}\left(1^{\prime}\right) a^{\dagger}\left(2^{\prime}\right) \ldots a^{\dagger}\left(n_{C}^{\prime}\right) a\left(n_{A}\right) \ldots a(2) a(1)
$$

of class [C.A] embodies, at least, one substructure $\in[k .0](k=1,2 \ldots)$ or/and $[k .1](k=2,3, \ldots)$. In this context, all primary Yukawa-type (trilinear) couplings should be eliminated from the interaction $V(\alpha)$ that enters

$$
H_{I}(\alpha)=V(\alpha)+\text { mass and vertex counterterms. }
$$

\footnotetext{
${ }^{1}$ A label $n$ is associated with all the necessary quantum numbers for a single particle
} 
It results in the form

$$
H=K_{F}\left(\alpha_{c}\right)+K_{I}\left(\alpha_{c}\right)=K,
$$

where the free part $K_{F}\left(\alpha_{c}\right)=H_{F}\left(\alpha_{c}\right)$ while the operator $K_{I}\left(\alpha_{c}\right)$ contains interactions between clothed particles. By construction, the latter has the property

$$
K_{I}\left(\alpha_{c}\right)|\Omega\rangle=K_{I}\left(\alpha_{c}\right)|n\rangle_{c} \equiv 0 .
$$

For the boson-fermion (meson-nucleon, photon-electron) system we have the decomposition

$$
\begin{aligned}
K_{I}\left(\alpha_{c}\right) & =K(f f \rightarrow f f)+K(\bar{f} \bar{f} \rightarrow \bar{f} \bar{f})+K(f \bar{f} \rightarrow f \bar{f})+K(b f \rightarrow b f)+K(b \bar{f} \rightarrow b \bar{f}) \\
& +K\left(f \bar{f} \leftrightarrow b b^{\prime}\right)+K(f f \leftrightarrow b f f)+K(f \bar{f} \leftrightarrow 3 b)+K(3 f \rightarrow 3 f)+\cdots,
\end{aligned}
$$

where separate contributions are responsible for different physical processes so, for instance, operators $K(\gamma e \rightarrow \gamma e), K(e e \leftrightarrow \gamma e e)$ and $K(3 N \rightarrow 3 N)$ can be used in describing the Compton scattering on electrons, the electron-electron bremsstrahlung and modeling three-nucleon forces, respectively. In particular, the fermion-fermion interaction operator in the CPR can be written as

$$
\begin{gathered}
K(f f \rightarrow f f)=\sum_{b} K_{b}(f f \rightarrow f f), \\
K_{b}(f f \rightarrow f f)=\int \sum_{\mu} d \vec{p}_{1}^{\prime} d \vec{p}_{2}^{\prime} d \vec{p}_{1} d \vec{p}_{2} V_{b}\left(1^{\prime}, 2^{\prime} ; 1,2\right) b_{c}^{\dagger}\left(1^{\prime}\right) b_{c}^{\dagger}\left(2^{\prime}\right) b_{c}(1) b_{c}(2),
\end{gathered}
$$

where the symbol $\sum_{\mu}$ denotes the summation over fermion spin projections, $1=\left\{\vec{p}_{1}, \mu_{1}\right\}$, etc.

\section{Interactions of vector fields with other fields}

Starting from the Lagrangian density

$$
L(x)=-\frac{1}{4} V^{\mu v}(x) V_{\mu v}(x)+\frac{1}{2} m_{v}^{2} V^{\mu}(x) V_{\mu}(x)-J_{v}^{\mu}(x) V_{\mu}(x)
$$

for a real massive vector field $V_{\mu}$ with $V^{\mu v}=\partial^{\mu} V^{v}-\partial^{v} V^{\mu}$, that is coupled via the current $J_{v}^{\mu}$ to other fields (the corresponding "free-particle" terms are omitted). By definition, the current does not involve $V_{\mu}$ ! In the framework of the canonical formalism one gets (see, e.g., Sec. 7.5 in [四]) the interaction Hamiltonian in Dirac $(D)$ picture,

$$
V(t)=\int d \vec{x} V(x)=\int d \vec{x}\left[J_{v}^{\mu}(x) v_{\mu}(x)+\frac{1}{2 m_{v}^{2}}\left[J_{v}^{0}(x)\right]^{2}\right],
$$

where $\vec{v}(x)$ is the $D$-picture counterpart of the vector $\vec{V}(x)$ in the Heisenberg $(H)$ picture, while the quantity $v_{0}(x)$ is introduced in a special way when eliminating the auxiliary component $V_{0}(x)$. Its density $V(x) \equiv V(t, \vec{x})$ does not possess the property to be invariant with respect to the Poincaré group $\Pi$, viz.,

$$
U_{F}(\Lambda, a) V(x) U_{F}^{-1}(\Lambda, a)=V(\Lambda x+a),
$$

$\forall \Lambda \in L_{+}$and arbitrary space-time shifts $a=\left(a^{0}, \vec{a}\right)$ 
The correspondence $(\Lambda, a) \rightarrow U_{F}(\Lambda, a)$ between elements $(\Lambda, a) \in \Pi$ and unitary transformations $U_{F}(\Lambda, a)$ realizes an irreducible representation of $\Pi$ in the Hilbert space (to be definite) of bosonfermion states. Here $L_{+}$is the homogeneous (proper) orthochronous Lorentz group.

We encounter a similar situation in the theory of interacting $\rho-, \omega-$ meson $(\varphi)$ and nucleon $(\psi)$ fields, where the corresponding densities can be represented as (see Appendix A in [Q] ) with the interaction $V_{v}=V^{(1)}+V^{(2)}$,

$$
V^{(1)}=\int d \vec{x}\left[g_{v} \bar{\psi}(\vec{x}) \gamma_{\mu} \psi(\vec{x}) \varphi^{\mu}(\vec{x})+\frac{f_{v}}{4 m} \bar{\psi}(\vec{x}) \sigma_{\mu \nu} \psi(\vec{x}) \varphi^{\mu v}(\vec{x})\right]
$$

and

$$
V^{(2)}=\int d \vec{x}\left[\frac{g_{v}{ }^{2}}{2 m_{v}^{2}} \bar{\psi}(\vec{x}) \gamma_{0} \psi(\vec{x}) \bar{\psi}(\vec{x}) \gamma_{0} \psi(\vec{x})+\frac{f_{v}{ }^{2}}{4 m^{2}} \bar{\psi}(\vec{x}) \sigma_{0 i} \psi(\vec{x}) \bar{\psi}(\vec{x}) \sigma_{0 i} \psi(\vec{x})\right]
$$

\section{QED Hamiltonian in Coulomb gauge. Parallels}

In CG the interaction Hamiltonian of the spinor QED is given by (cf., for example, Eqs. (8.4.3) and (8.4.23) in [四])

$$
V_{\text {qed }}=\int d \vec{x} V_{q e d}(\vec{x})=\int d \vec{x} J^{k}(\vec{x}) a_{k}(\vec{x})+V_{\text {Coul }},
$$

with the electron-positron current $J^{\mu}(\vec{x})=e \bar{\psi}(\vec{x}) \gamma^{\mu} \psi(\vec{x})$ and the Coulomb part,

$$
V_{\text {Coul }}=\frac{1}{2} \int d \vec{x} \int d \vec{y} \frac{J^{0}(\vec{x}) J^{0}(\vec{y})}{4 \pi|\vec{x}-\vec{y}|} .
$$

Evidently, the corresponding interaction density $V_{\text {qed }}(x)$ is not Lorentz scalar. In this respect, for CG, where the photon field $A_{\mu}(\vec{x})$ is introduced in such a way to have $A_{0}(\vec{x}) \equiv 0$, a resemblance of the first terms in r.h.s. of Eqs. (4. (ل) and (3.2) is misleading. However, in both cases we cannot use the so-called Belinfante ansatz to construct the boost generator $\vec{N}$, i.e., put, for example,

$$
\mathbf{N}_{\text {qed }}=-\int \mathbf{x} V_{\text {qed }}(\vec{x}) d \vec{x} .
$$

Therefore one has to seek other ways to provide the relativistic invariance (RI) in Dirac sense (see, e.g., [[]]).

Further, by using the Fourier expansions

$$
\begin{aligned}
& v^{\mu}(\vec{x})=\int \frac{d \vec{k}}{\sqrt{2(2 \pi)^{3} \omega_{\vec{k}}}} \sum_{s}\left[e^{\mu}(\vec{k}, s) v(\vec{k}, s)+e^{\mu *}(-\vec{k}, s) v^{\dagger}(-\vec{k}, s)\right] \exp (\overrightarrow{i k} \vec{x}), \\
& \vec{a}(\vec{x})=\int \frac{d \vec{k}}{\sqrt{2(2 \pi)^{3}|\vec{k}|}} \sum_{\sigma}\left[\vec{e}(\vec{k}, \sigma) a(\vec{k}, \sigma)+\vec{e}^{*}(-\vec{k}, \sigma) a^{\dagger}(-\vec{k}, \sigma)\right] \exp (\overrightarrow{i k} \vec{x}), \\
& \psi(\vec{x})=\int d \vec{p} \sqrt{\frac{m}{(2 \pi)^{3} E_{\vec{p}}}} \sum_{\mu}\left[\vec{u}(\vec{p} \mu) b(\vec{p} \mu)+v(-\vec{p} \mu) d^{\dagger}(-\vec{p} \mu)\right] \exp (i \vec{p} \vec{x}),
\end{aligned}
$$

where $E_{\vec{p}}=\sqrt{\vec{p}^{2}+m^{2}}\left(\omega_{\vec{k}}=\sqrt{\vec{k}^{2}+m_{v}^{2}}\right)$ is the fermion (boson) energy, one can express the Hamiltonians (other generators of the Poincare group, currents, etc.) through the creation and annihilation 
operators which, satisfying canonical commutation and anticommutation rules, compose the set $\alpha$. It is implied that the quantities are normally ordered with respect to these operators.

After this, following the prescription given above, we perform the first clothing transformation $W^{(1)}=\exp \left[R^{(1)}\right]\left(R^{(1)^{\dagger}}=-R^{(1)}\right)$, which eliminates the primary interactions $V^{(1)}$ in the first order in coupling constants assuming that these $V^{(1)}$ consist of bad terms only.

In the case under consideration we have interactions in $\mathrm{D}$ picture

$$
V^{(1)}(t)=\int d \vec{x} V^{(1)}(x) \equiv \int d \vec{x} J_{v}^{\mu}(x) v_{\mu}(x)
$$

and

$$
V_{q e d}^{(1)}(t)=\int d \vec{x} V_{q e d}^{(1)}(x) \equiv \int d \vec{x} J^{\mu}(x) a_{\mu}(x)
$$

that are trilinear in the creation and annihilation operators involved.

Operator $R^{(1)}$ obeys the equation for its finding

$$
\left[R^{(1)}, H_{F}\right]+V^{(1)}=0
$$

which has the solution

$$
R^{(1)}=-i \lim _{\varepsilon \rightarrow 0+} \int_{0}^{\infty} V_{D}^{(1)}(t) e^{-\varepsilon t} d t
$$

if $m_{v}<2 m$. Evidently, this inequality is valid with $m_{v}=m_{\gamma}$ and $m=m_{e^{+}}=m_{e^{-}}=m_{e}$. One should stress that from this moment all bare-particle operators $\alpha$ are replaced by clothed-particle counterparts $\alpha_{c}$.

For this presentation I will confine myself to the consideration of $2 \rightarrow 2$ processes (in particular, electron-electron scattering). The corresponding interaction operator $V(e e \rightarrow e e)$ in $\mathrm{CPR}$ is determined by the first term in the r.h.s. of

$$
\begin{aligned}
& K_{I}\left(\alpha_{c}\right)=K\left(e^{-} e^{-} \rightarrow e^{-} e^{-}\right)+K\left(e^{+} e^{+} \rightarrow e^{+} e^{+}\right)+K\left(e^{+} e^{-} \rightarrow e^{+} e^{-}\right) \\
& +K\left(\gamma e^{ \pm} \rightarrow \gamma e^{ \pm}\right)+K\left(e^{+} e^{-} \leftrightarrow \gamma+\gamma\right)+K\left(e^{-} e^{-} \leftrightarrow \gamma e^{-} e^{-}\right)+\cdots
\end{aligned}
$$

that yields in $e^{2}$ order

$$
V(e e \rightarrow e e) \equiv K^{(2)}\left(e^{-} e^{-} \rightarrow e^{-} e^{-}\right)=\frac{1}{2}\left[R^{(1)}, V^{(1)}\right]\left(e^{-} e^{-} \rightarrow e^{-} e^{-}\right)+V_{\text {Coul }}\left(e^{-} e^{-} \rightarrow e^{-} e^{-}\right)
$$

and after a simple algebra we get for two clothed electrons

$$
\begin{gathered}
\frac{1}{2}\left[R^{(1)}, V^{(1)}\right]\left(e^{-} e^{-} \rightarrow e^{-} e^{-}\right)=V_{e e}-V_{C o u l}(e e), \\
V_{e e} \equiv K_{\gamma}(e e \rightarrow e e)=\int \sum_{\mu} d \vec{p}_{1}^{\prime} d \vec{p}_{2}^{\prime} d \vec{p}_{1} d \vec{p}_{2} V_{\gamma}\left(1^{\prime}, 2^{\prime} ; 1,2\right) b^{\dagger}\left(1^{\prime}\right) b^{\dagger}\left(2^{\prime}\right) b(1) b(2)
\end{gathered}
$$

with $c$-number matrix

$$
\begin{gathered}
V_{\gamma}\left(1^{\prime}, 2^{\prime} ; 1,2\right)=\frac{e^{2}}{(2 \pi)^{3}} \frac{m_{e}^{2}}{\sqrt{E_{\vec{p}_{1}^{\prime}} E_{\vec{p}_{2}^{\prime}} E_{\vec{p}_{1}} E_{\vec{p}_{2}}}} \delta\left(\vec{p}_{1}^{\prime}+\vec{p}_{2}^{\prime}-\vec{p}_{1}-\vec{p}_{2}\right) v\left(1^{\prime}, 2^{\prime} ; 1,2\right), \\
v\left(1^{\prime}, 2^{\prime} ; 1,2\right)=\frac{1}{2} \frac{\bar{u}\left(\vec{p}_{1}^{\prime}\right) \gamma^{\mu} u\left(\vec{p}_{1}\right) \bar{u}\left(\vec{p}_{2}^{\prime}\right) \gamma_{\mu} u\left(\vec{p}_{2}\right)}{\left(p_{1}-p_{1}^{\prime}\right)^{2}-m_{\gamma^{2}}}
\end{gathered}
$$


When deriving these formulae we have used the completeness condition for photon polarizations and the representation,

$$
\begin{gathered}
V_{\text {Coul }}\left(e^{-} e^{-} \rightarrow e^{-} e^{-}\right) \equiv V_{\text {Coul }}(e e)=\int \sum_{\mu} d \vec{p}_{1}^{\prime} d \vec{p}_{2}^{\prime} d \vec{p}_{1} d \vec{p}_{2} V_{\text {Coul }}\left(1^{\prime}, 2^{\prime} ; 1,2\right) b^{\dagger}\left(1^{\prime}\right) b^{\dagger}\left(2^{\prime}\right) b(1) b(2), \\
V_{\text {Coul }}\left(1^{\prime}, 2^{\prime} ; 1,2\right)=\frac{e^{2}}{(2 \pi)^{3}} \frac{m_{e}^{2}}{\sqrt{E_{\vec{p}_{1}^{\prime}} E_{\vec{p}_{2}^{\prime}} E_{\vec{p}_{1}} E_{\vec{p}_{2}}}} \delta\left(\vec{p}_{1}^{\prime}+\vec{p}_{2}^{\prime}-\vec{p}_{1}-\vec{p}_{2}\right) v_{\text {Coul }}\left(1^{\prime}, 2^{\prime} ; 1,2\right), \\
v_{\text {Coul }}\left(1^{\prime}, 2^{\prime} ; 1,2\right)=-\frac{1}{2} \frac{\bar{u}\left(\vec{p}_{1}^{\prime}\right) \gamma^{0} u\left(\vec{p}_{1}\right) \bar{u}\left(\vec{p}_{2}^{\prime}\right) \gamma^{0} u\left(\vec{p}_{2}\right)}{\left(\vec{p}_{1}-\vec{p}_{1}^{\prime}\right)^{2}} .
\end{gathered}
$$

In order to preserve the continuity with the vector-meson-nucleon interactions we do not hurry to put $m_{\gamma}=0$. Besides, keeping in mind the problem of removing the infrared divergences sometimes it is convenient to handle an infinitesimally small photon mass.

It is time to quote from [四] on p. 355, viz., " ... the apparent violation of Lorentz invariance in the instantaneous Coulomb interaction cancels by another apparent violation of Lorentz invariance ..." that arises since the photon operators $A_{\mu}(x)$ do not make a four-vector. An important point is that in CPR, unlike [四], such a cancellation (cf. our results [ [ $]$ in mesodynamics ) takes place directly in the Hamiltonian .

Such a distinct feature of the UCT method makes it useful for covariant calculations of the $S$ matrix either by solving the two-particle Lippmann-Schwinger equation (LSE) for the corresponding $T$-matrix or using the perturbation theory (not obligatorily addressing the Dyson-Feynman expansion). In this context, I would like to note an akin approach to problems of relativistic QFT, developed in [10]].

Of course, doing so one can find not only the $S$-matrix but the eigenstates of operator $K=K_{F}+V_{e e}$ in the Fock subspace $H_{F}^{[2]}$ spanned onto the clothed-two-particle $K_{F}$ eigenvectors. In this connection, one has to deal with

$$
K_{F}=\int d \vec{k} \omega_{\vec{k}} \sum_{\sigma} a^{\dagger}(\vec{k} \sigma) a(\vec{k} \sigma)+\int d \vec{p} E_{\vec{p}} \sum_{\mu}\left[b^{\dagger}(\vec{p}, \mu) b(\vec{p}, \mu)+d^{\dagger}(\vec{p}, \mu) d(\vec{p}, \mu)\right]
$$

omitting, for brevity, the lower index $c$ at operators in the r.h.s. of this expression.

\section{QCD Hamiltonian in CG. Some Similarities}

At last, I would like to drawing some parallels between QED and QCD, where we find (see, e.g., survey [ए]] ) the QCD Lagrangian density

$$
L_{q c d}(x)=-\frac{1}{4} F_{a}^{\mu v}(x) F_{\mu v}^{a}(x)+\frac{1}{2}\left[\overline{\tilde{\Psi}}(x)\left(i \gamma_{\mu} \tilde{D}^{\mu}-\tilde{m}\right) \tilde{\Psi}(x)+\text { h.c. }\right]
$$

with tensor of color-electro-magnetic fields $F_{a}^{\mu v}=\partial^{\mu} A_{a}^{v}-\partial^{v} A_{a}^{\mu}+g\left(A^{\mu} \times A^{v}\right)_{a}{ }^{2}$ (gluon index $a(r s)$ from 1 to $\left.n_{c}^{2}-1\right)$, color vector potentials $A_{a}^{\mu}$ (gluon-field components), color masses $\tilde{m}_{c c^{\prime}}=$ $\tilde{m} \delta_{c c^{\prime}}$ and covariant color derivatives $\tilde{D}_{c c^{\prime}}^{\mu}=\delta_{c c^{\prime}} \partial^{\mu}-i g \tilde{A}_{c c^{\prime}}^{\mu}$, these $n_{c} \otimes n_{c}$ matrices (color indices

\footnotetext{
${ }^{2}$ For the two matrices $A$ and $B$, by definition, the composition $(A \times B)_{a}=i f^{a r s} A_{r} B_{s}$ with the structure constants $f^{\text {ars. }}$.
} 
$c$ and $c^{\prime}$ run from 1 to $\left.n_{c}\right)$, color-Maxwell equations $\partial_{v} F_{a}^{\mu v}=g J_{a}^{\mu}$ and color-Dirac ones $\left(i \gamma_{\mu} \tilde{D}^{\mu}-\right.$ $\tilde{m}) \tilde{\Psi}=0$ for quark fields $q(x) \equiv \tilde{\Psi}(x)$ in case of the $S U(3)$ gauge model with conserved color currents $J_{a}^{\mu}=\tilde{\tilde{\Psi}} \gamma^{\mu} T_{a} \tilde{\Psi}+f^{\text {ars }} F_{r}^{\mu \lambda} A_{\lambda}^{s}$, the matrices $T_{a}$ act in color space and for $S U(3)$ are related to the Gell-Mann matrices $T_{a}=\frac{1}{2} \lambda_{a}$

versus

the QED Lagrangian density

$$
L_{q e d}(x)=-\frac{1}{4} F^{\mu v}(x) F_{\mu v}(x)+\frac{1}{2}\left[\bar{\Psi}(x)\left(i \gamma_{\mu} D^{\mu}-m\right) \Psi(x)+\text { h.c. }\right]
$$

with the Maxwell equations $\partial_{v} F^{\mu v}=g J^{\mu}(g=e$ !) and the conserved electron-positron current $J^{v}=\bar{\Psi} \gamma^{\nu} \Psi$ and the Dirac equations $\left(i \gamma_{\mu} D^{\mu}-m\right) \Psi=0$ with the conventional $D^{\mu}=\partial^{\mu}-i e A^{\mu}$.

Going on, one gets color energy-momentum four-vector

$$
P_{q c d}^{v}=\int d \vec{x}\left(F_{a}^{0 \lambda} F_{a, \lambda}^{v}-g^{0 v} L_{q c d}+\frac{1}{2}\left[i \bar{q} \gamma_{0} \tilde{D}^{v} q+\text { h.c. }\right]\right)
$$

versus

$$
P_{q e d}^{v}=\int d \vec{x}\left(F^{0 \lambda} F_{\lambda}^{v}-g^{0 v} L_{q e d}+\frac{1}{2}\left[i \bar{\psi} \gamma_{0} D^{v} \psi+\text { h.c. }\right]\right) .
$$

All these quantities are gauge invariant, i.e., remain unchanged with respect to the simultaneous transformations

$\psi \Rightarrow \psi^{\prime}=U \psi, A_{\mu} \Rightarrow A_{\mu}^{\prime}=U A_{\mu} U^{\dagger}-i g^{-1}\left(\partial_{\mu} U\right) U^{\dagger}$ with unitary matrix operator $U=\exp (-i g \varepsilon(x))$ (of course, in color space for such a non-abelian gauge theory as QCD).

The corresponding Hamiltonians are

$$
P_{q c d}^{0}=\int d \vec{x}\left(F_{a}^{0 \lambda} F_{a, \lambda}^{0}-L_{q c d}+\frac{1}{2}\left[i \bar{q} \gamma_{0} \tilde{D}^{0} q+\text { h.c. }\right]\right)
$$

and

$$
P_{q e d}^{0}=\int d \vec{x}\left(F^{0 \lambda} F_{\lambda}^{0}-L_{q e d}+\frac{1}{2}\left[i \bar{\psi} \gamma_{0} D^{0} \psi+\text { h.c. }\right]\right) .
$$

From practical point of view it is convenient to employ CG in which $\partial^{k} A_{k}^{a}=0$ so that Gauss' law is satisfied for each of the gluon fields involved. In particular, it is the case where the color-Maxwell equations can be divided into the equations of motion

$$
\partial_{v} F_{a}^{k v}=g J_{a}^{k}=g \bar{q} \gamma^{k} T_{a} q+i g\left(A_{\lambda} \times F^{k \lambda}\right)_{a}
$$

and the equations of constraint

$$
\partial_{k} F_{a}^{0 k}=g J_{a}^{0}=g \bar{q} \gamma^{0} T_{a} q+i g\left(A_{\lambda} \times F^{0 \lambda}\right)_{a} .
$$

Further, one can choose the transverse gauge potentials $A_{k}^{a}$ and the transverse color electric fields $E_{i}^{a T}=F_{0 i}^{a T}=F_{0 i}^{a}-F_{0 i}^{a L}=E_{i}^{a}-E_{i}^{a L}$ as the independent variables, where the longitudinal fields $E_{i}^{a L}$ are determined from the constraint relation (5.8) that yields

$$
\partial^{k} F_{k 0}^{a L}+g\left(A^{k} \times F_{k 0}^{L}\right)_{a}=-j_{a}^{0}+g\left(E_{k} \times A^{k}\right)_{a} .
$$

In terms of these variables the QCD Hamiltonian takes the form

$$
P_{q c d}^{0}=H_{q c d}=\int d \vec{x} \frac{1}{2}\left[\bar{q}\left(i \gamma_{k} \partial^{k}-m\right) q+\text { h.c. }\right]+\frac{1}{2}\left(F_{k 0}^{a T} F_{k 0}^{a T}+\vec{B}_{a} \vec{B}_{a}\right)+\frac{1}{2} F_{k 0}^{a L} F_{k 0}^{a L}
$$




$$
+V_{\text {vertex }}+\text { mass counterterms }
$$

with the so-called vertex quark-gluon interaction

$$
V_{\text {vertex }}=g \int d \vec{x} j_{a}^{k}(\vec{x}) A_{k}^{a}(\vec{x})
$$

where $j_{\mu}^{a}=\bar{q} \gamma_{\mu} T_{a} q$ is the quark - antiquark current.

Again in the Fock representation we have addressed the corresponding set $\alpha$ from

$$
\begin{aligned}
& \vec{A}_{a}(\vec{x})=\int \frac{d \vec{k}}{\sqrt{2(2 \pi)^{3}|\vec{k}|}} \sum_{\sigma}\left[\vec{e}(\vec{k}, \sigma) A_{a}(\vec{k}, \sigma)+\vec{e}^{*}(-\vec{k}, \sigma) A_{a}^{\dagger}(-\vec{k}, \sigma)\right] \exp (\overrightarrow{i k} \vec{x}), \\
& q_{f c}(\vec{x})=\int d \vec{p} \sqrt{\frac{m}{(2 \pi)^{3} E_{\vec{p}}}} \sum_{\mu}\left[\vec{u}(\vec{p} \mu) b_{f c}(\vec{p} \mu)+v(-\vec{p} \mu) d_{f c}^{\dagger}(-\vec{p} \mu)\right] \exp (i \vec{p} \vec{x})
\end{aligned}
$$

with the flavor-color label $f c$, perhaps, and the canonical commutations

$$
\begin{gathered}
{\left[A_{a}(\vec{k}, \sigma), A_{a^{\prime}}^{\dagger}\left(\vec{k}^{\prime}, \sigma^{\prime}\right)\right]_{+}=\delta\left(\vec{k}-\vec{k}^{\prime}\right) \delta_{\sigma \sigma^{\prime}} \delta_{a a^{\prime}},} \\
{\left[b_{f c}(\vec{p} \mu), b_{f^{\prime} c^{\prime}}^{\dagger}\left(\vec{p}^{\prime} \mu^{\prime}\right)\right]_{-}=\left[d_{f c}(\vec{p} \mu), d_{f^{\prime} c^{\prime}}^{\dagger}\left(\vec{p}^{\prime} \mu^{\prime}\right)\right]_{-}=\delta\left(\vec{p}-\vec{p}^{\prime}\right) \delta_{\mu \mu^{\prime}} \delta_{f f^{\prime}} \delta_{c c^{\prime}}}
\end{gathered}
$$

to start with the clothing procedure exposed above. Doing so to build a novel family of interactions between the clothed quarks and gluons we will show to what extent the $R$ - commutators will cancel some counterparts that stem from the third term in the r.h.s. of Eq. (Б) and those hidden in the renormalization box there. This research is under way.

\section{To Conclude}

- Using the instant form of relativistic dynamics and relying upon our previous experience we show applications of the UCT method for popular field models of interacting mesons and nucleons, photons and electrons, gluons and quarks, etc.

- Mass-changing Bogoliubov-type transformations lead to the bare particles with given mass values

- Within our approach the two-clothed-electron interaction $V_{e e}$ gets the covariant form due to cancellation of the non-covariant primary Coulomb interaction contribution to the QED Hamiltonian. An important point is that in the CPR, unlike [四], such a cancellation (cf. our results [9] in mesodynamics ) takes place directly in the Hamiltonian

- Trying to realize the notion of clothing in QCD, we start from the well-known QCD Lagrangian density with the hermitian and traceless vector potentials, mass and covariant derivative matrices in color space, color-Maxwell equations and color gauge-invariant energymomentum stress tensor versus their colorless counterparts in QED. Of course, a long way lies ahead. 
- A novel family of relativistic hermitean and energy-independent interactions can be built with help of the UCT method for each of these systems

- In addition, I'd like to stress: the clothing procedure opens a fresh look at calculations of mass and charge shifts.

\section{References}

[1] S. Weinberg, The Quantum Theory of Fields Vol. I, Cambridge university press,1995).

[2] A. Shebeko, M. Shirokov, Clothing procedure in relativistic quantum field theory and its applications to description of electromagnetic interactions with nuclei (bound systems), Progr. Part. Nucl. Phys. 44 (2000) 75.

[3] A. V. Shebeko, M. I. Shirokov, Unitary transformations in quantum field theory and bound states, Phys. Part. Nuclei 32 (2001) 31 arXiv:nucl-th/0102037.

[4] V. Korda, A. Shebeko, Clothed Particle Representation in Quantum Field Theory: Mass Renormalization, Phys. Rev. D70 (2004).

[5] V. Korda, L. Canton, A. Shebeko, Relativistic interactions for the meson-two-nucleon system in the clothed-particle unitary representation, Ann. Phys. 322 (2007) 736 arXiv:nucl-th/0603025

[6] N.N. Bogoliubov, V.V. Tolmachev and D.V. Shirkov, A New Method in the Theory of Superconductivity, Consultants Bureau (New York, 1959).

[7] A. V. Shebeko in: Advances in Quantum Field Theory, Ed. S. Ketov, INTECH (Rijeka,2012), pp. 3 -30 .

[8] A. V. Shebeko, P. A. Frolov, A possible way for constructing the generators of the Poincaré group in quantum field theory, Few Body Syst. 52 (2012) 125 arXiv:1107.5877 [hep-th].

[9] I. Dubovyk, A. Shebeko, The method of unitary clothing transformations in the theory of nucleon-nucleon scattering, Few-Body Syst. 48 (2010) 109.

[10] E. Stefanovich, Quantum field theories without infinities, Ann. Phys., 292 (2010) 139.

[11] S. J. Brodsky, H.-Ch. Pauli and S.S. Pinsky, Quantum chromodynamics and other field theories on the light cone, Phys. Rep. 301 (1998) 299. 